\title{
A SOLUTION OF SUBORDINATE VERTICES FOR QUALITY CONNECTIONS OF EXTERNAL FREE-FORM CONCRETE PANELS
}

\author{
Jeeyoung LIM$^{1}$, Seunghyun SON${ }^{1}$, Do Yeong KIM${ }^{1}$, Heni FITRIANI², Sunkuk KIM ${ }^{1 *}$ \\ ${ }^{1}$ Department of Architectural Engineering, Kyunghee University, 1732 Deogyeong-daero, 17104, \\ Yongin-si, Republic of Korea \\ ${ }^{2}$ Department of Civil Engineering, Universitas Sriwijaya, Palembang, Sumatera Selatan, Indonesia
}

Received 04 May 2018; accepted 21 June 2018

\begin{abstract}
D-designs of free-form buildings are developed using a computer due to difficulty of shape implementation. When producing free-form concrete panel (FCP) using materials such as GFRC (glass fiber reinforced concrete), engineers or manufacturers should precisely calculate the offset value or geometry of each member at the junction point of three or more FCPs before it is constructed. However, it is difficult to calculate offset geometry easily and quickly, and no research has been conducted on this topic. Therefore, the objective of this paper is to develop a solution of subordinate vertices for quality connections of external free-form concrete panels. The developed mathematical solutions practically support the production of FCPs with precise installation to ensure aesthetic quality of the building. This paper academically contributes to the automatic creation of joint details of FCPs implemented by BIM.
\end{abstract}

Keywords: free-form, concrete panel, subordinate vertex, offset geometry, quality connection.

\section{Introduction}

To design beautiful free-form buildings, many architects use BIM (building information modeling) tools such as Rhino and CATIA (computer aided three dimensional interactive application), which can easily express and transform free-form shapes (Ryu 2012; Ryu, Kim 2012). The finish pattern of the designed free-form building is divided into aesthetics (Lee et al. 2015) and is composed of free-form panels at the production and installation stages (Lee et al. 2015; Kim et al. 2015; Kim 2014).

For free-form panels, various materials such as glass, aluminium, titanium, UHPC (ultra-high performance concrete), and GFRC (glass fiber reinforced concrete) have been used in addition to steel and concrete (Kim et al. 2015). Among them, metal is a representative material of free-form building members, but it has high material cost and high production cost. In addition, there is a risk of deformation due to impact and corrosion. In contrast, concrete is excellent in heat insulation, formability and strength. It is easy to maintain and can solve the problems of metal materials. However, general concrete is difficult to use as an exterior panel because it is heavy and lacks the expressiveness of free-form shaped metal materials. Also, general concrete has a low strength even though it is heavy.
To solve this problem, FCP (free-form concrete panel) production technologies using materials such as UHPC and GFRC have been developed and are being studied to improve production efficiency, installation, and maintenance (Son et al. 2018).

The free-form roof of the Qatar national museum consists of more than 15,000 GFRC panels, all of which have different sizes and shapes (Kim 2014). GFRC improves and reinforces the mechanical properties of mortar or concrete and is superior to general concrete in terms of bending strength, tensile strength, impact resistance, airtightness, and water tightness (Kim et al. 2008; Daniel, Shah 1990; Peled et al. 2000; Precast/Pre-stressed Concrete Institute 2001). Glass curtain walls and UHPC were used as exterior materials for the Louis Vuitton Foundation for Creation, and about 18,800 FCPs were produced in different shapes. The UHPC used has excellent structural stability due to its compressive strength of $200 \mathrm{MPa}$ and its own tensile strength (Kim 2010; Reda et al. 1999).

It is not easy for each member of multiple FCPs with a certain thickness to meet exactly at one point during production-installation. In other words, accurate dimensions and geometries should be provided at the design

*Corresponding author. E-mail: kimskuk@khu.ac.kr 


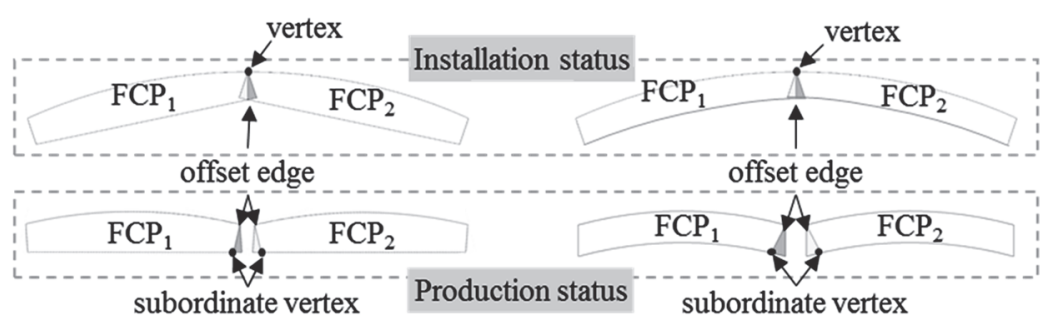

(a) Offset joint detail of one-side FCPs

(b) Offset joint detail of two-side FCPs

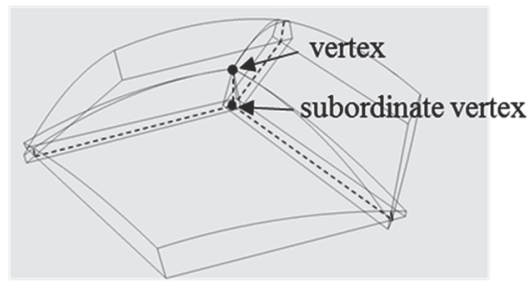

(c) Vertices with three FCPs

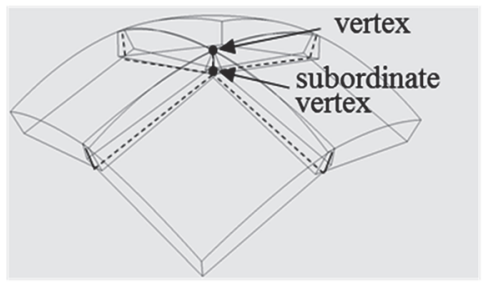

(d) Vertices with four FCPs

Figure 1. Vertices formed by FCPs

stage to precisely match a vertex that meets at the top of a member and a subordinate vertex that meets at the bottom, as shown in Figure 1. The subordinate vertices are points that occur when two or more members meet at different angles, and the edges are cancelled by considering the thickness of each member (Kim 2014). If these vertices are considered during the production of members, it is easy to perform assembly and installation on-site. Offset joint details of one-sided FCPs can be expressed as shown in Figure 1(a), and offset joint details of two-sides FCPs are shown in Figure 1(b). As shown in Figure 1(c) and Figure $1(\mathrm{~d})$, the number of subordinate vertices increases and forms a complex shape when three or more FCPs meet.

That is, if two or more FCPs meet at different angles, the end side of FCPs must be offset for precise connections. It is necessary to precisely calculate the offset value or geometry of each member at the junction point of three or more FCPs before the member can be installed precisely. Therefore, the objective of this paper is to develop a solution of subordinate vertices for quality connections of external free-form concrete panels.

This study is conducted in the following four stages:

1. Examine the necessity to solve vertex problems using BIM in designing FCPs based on a literature review.

2. When assembling three or more FCPs, the members must be exactly aligned at the subordinate vertex. For this, define mathematically the position of the subordinate vertex, which is the reference point for calculating the offset volume of each member.

3. Derive a formula that defines the location of the subordinate vertex corresponding to the number of FCPs encountered at a point.

4. Create mathematical algorithms to calculate the offset volume using the equations defined.

5. Select the case project, apply the derived algorithms, and discuss the effectiveness of these algorithms.

\section{Preliminary study}

Studies on free-form concrete panels have been emerging for many decades, and these are mostly based on an analysis of the design process of the building, the morphological characteristics, and the concept of the design technology from a design point of view. Bae et al. (2009) presented a method for digital design by analyzing the background and showed a case where the digital design process was applied to free-form architecture. Lyu (2009) analyzed the background and a case where the digital design process was used to implement free-formed architecture based on five criteria: Sectioning, Tessellating, Folding, Contouring, and Forming. Jovanovic et al. (2017) mainly focused on the use of Grasshopper 3D/Rhino for digital designs and proposed comprehensive tessellation algorithm approaches for numerous design strategies. This research also mentioned that Rhino was most commonly applied for design-based processes (form generation, rationalization, and tessellation). Sacks et al. (2004) developed parametric 3D modeling of precast concrete to improve productivity during the design process. This paper also discussed technical problems by reviewing the concepts, features, and applications of such concrete. Ryu and Kim (2013) discussed digital design optimization considering the workability of free-formed structures. Those previous studies specifically focused on the design process of FCPs.

Many architects use a parametric modeling approach as a digital design tool that allows them to easily and simultaneously create and modify the design patterns. In the design stage, the use of BIM technologies such as Rhino and CATIA has played significant roles in actualizing free formed buildings both for aesthetic purposes and for production and installation of the finished panel design (FCPs). Therefore, it is necessary to ensure the stability of the joined surfaces, called subordinate vertices, among connected FCPs. Modifying joined segments to fit 


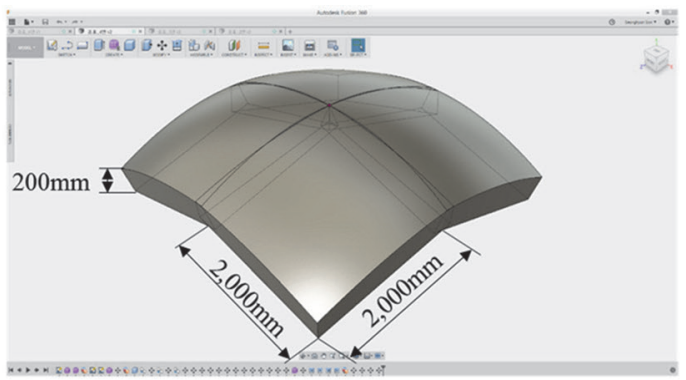

(a) Connection with four FCPs

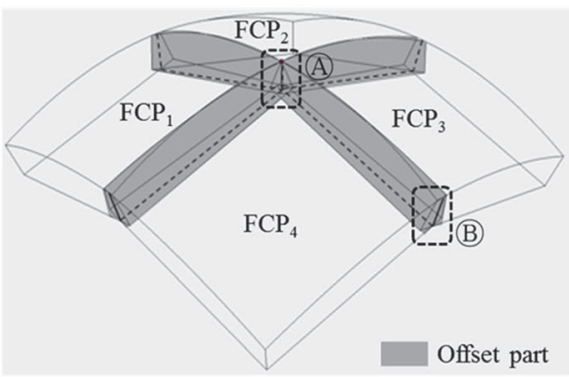

(b) Offset joint part of design sample

Figure 2. Design sample with four FCPs

the design may reduce workload, time, and cost. Keneti et al. (2008) developed a new algorithm to identify the 3D contact patterns and associated contacts between convex blocks. This study proposed the theory of segment intersections using global coordination systems. However, it was limited only to convex blocks and did not address concave contact shapes. Kulovec et al. (2012) suggested a postoptimization procedure by achieving the minimum joinheight differences for all joints of a free-form architectural design meshed with a quad-dominant mesh. However, if there is edge contact with each neighboring panel along the thickness, the panels must be cut at different angles, causing differences in the heights. This paper considered the use of spacers of different thickness to compensate for the residual height differences. Li et al. (2010) studied the possibility of controlling the type, location, and number of free-formed vertices of a triangle mesh. Although these studies emphasized the importance of design finish patterns, there is no practical application that can identify issues and solve problems at the vertex point of FCPs during the design process.

Due to difficulties during the production of precise FCPs, it is essential to be accurate in producing joints before FCP installation without cutting the segments with a computerized numeric control (CNC) machine (Lim et al. 2018). The vertex point is the corner vertex occurring when two or more members meet at different angles. For this reason, research on free-formed architecture remains at a very basic level, mainly including case studies, problem analysis, and design process proposals, and there is no recognition of the problem of vertex marking during external installation using FCPs for free-form buildings. Previous studies have not yet considered vertex points for connecting external free-form concrete panels. The development of technology in computer science has made it possible to design free-form concrete panels with freeformed shapes for each panel. Problems associated with joining details for a number of panels connected to each other can be diminished during the design process. However, these joints must match for proper installation. Thus, the joining details for connecting a number of FCPs must be studied to develop a more productive and precise installation and to develop a vertex formula for minimizing the work and manpower input during production and installation of the designed FCPs.

\section{Mathematical definition of subordinate vertices}

\subsection{Analysis of subordinate vertex}

Considering the finish pattern, erection and installation may be easier on site when the joint points of FCPs are offset. However, when connecting the produced FCPs based on the vertex at the upper section, they can be offset by as much as the panel thickness. For instance, a subordinate vertex and offset joint parts are generated when connecting four FCPs as shown in Figure 2. A 3D modeling tool (Fusion 360) was used. As shown in Figure 2(a), the designed sample has a square shape $200 \mathrm{~mm}$ thick and 2,000 $\mathrm{mm}$ in length/width. This is expressed in $\mathrm{x}$-ray mode, and the offset part was expressed in grey in Figure 2(b).

Looking closer at (A) of Figure 2, the connection of the four FCPs is as shown in Figure 3. When the upper corner edges of $F C P_{1}, F C P_{2}, F C P_{3}$, and $F C P_{4}$ contact $O$, the lower section of each panel is offset. When each panel edge is offset, the corner edges $\left(a_{1}, a_{2}, a_{3}\right.$, and $\left.a_{4}\right)$ of each panel are in contact with the subordinate vertex $\left(\mathrm{O}^{\prime}\right)$. For $F C P_{1}$, the volume between the corner edge $\left(a_{1}\right)$ and subordinate vertex $\left(O^{\prime}\right)$ should be offset. The same applies (i) for $\mathrm{FCP}_{2}$, which is between $a_{2}$ and $O^{\prime}$; (ii) for $F C P_{3}$, which is between $a_{3}$ and $O^{\prime}$; and (iii) for $\mathrm{FCP}_{4}$, which is between $a_{4}$ and $O^{\prime}$. In other words, the offset parts should be cut at the end of the production stage so that FCPs can be installed as finished panels.

\subsection{Mathematical concept of subordinate vertices}

Examining section (B) in Figure 2(b), there is an offset part as shown in Figure 4(a). In $\mathrm{FCP}_{4}$ and $\mathrm{FCP}_{3}$, the offset part (C) shown in Figure 3(b) is generated when panels are connected based on thickness and curvature. The offset angles of $F C P_{3}$ and $F C P_{4}$ are defined as $d \theta_{1}$ and $d \theta_{2}$, respectively, and the offset lengths are $w_{34}$ and $w_{43} . d \theta_{1}$ is calculated as shown in Eqn (1), and $d \theta_{2}$ can be calculated in the same manner.

$$
d \theta_{1}=\tan ^{-1}\left(\frac{w_{1}}{t}\right) .
$$

If the thickness and curvature of both panels are the same, $w_{1}=w_{2}$ and $d \theta_{1}=d \theta_{2}$. Eqn (2) is used to define the curvature of a line, as shown in Figure 5. The offset length $\Delta s$ is equivalent to the sum of $w_{1}$ and $w_{2}$, as shown in Eqn (3). That is, the geometry of offset parts can be estimated using the curvature, thickness, and FCP coordinates. 


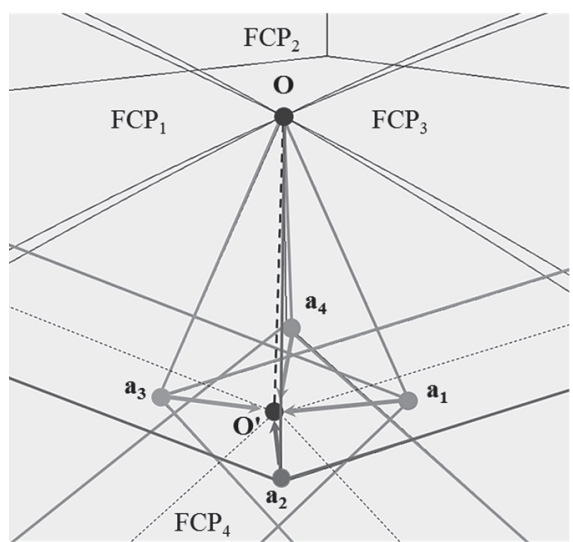

$\mathrm{a}_{1}, \mathrm{a}_{2}, \mathrm{a}_{3}, \mathrm{a}_{4}$ : point of corner edge of $\mathrm{FCP}_{1}$, $\mathrm{FCP}_{2}, \mathrm{FCP}_{3}, \mathrm{FCP}_{4}$

$\mathrm{O}:$ vertex

$\mathrm{O}^{\prime}$ : subordinate vertex

Figure 3. Vertices with four FCPs (A)

$\Delta s=\rho \cdot \Delta \theta \cong \sqrt{\Delta x^{2}+\Delta y^{2}}$

$\rho=\lim _{\Delta x \rightarrow 0} \frac{\Delta s}{\Delta \theta}=\cdots=\frac{\left[1+\left(\frac{d y}{d x}\right)^{2}\right]^{\frac{3}{2}}}{\frac{d^{2} y}{d x^{2}}} ;$

$\Delta \theta=\frac{\Delta s}{\rho}$

$2 \cdot \Delta s=w_{1}+w_{2}$.

\subsection{Calculation algorithms of angles}

(1) Subordinate vertex with three-sided FCPs

As shown in Figure 6(a), the bottom of panel FCP without considering offset volume consists of $a_{1}, b_{1}, c_{1}$, and $d_{1}$. The same applies for (i) $F C P_{2}$, which is $a_{2}, b_{2}, c_{2}$, and $d_{2}$; (ii) for $F C P_{3}$, which is $a_{3}, b_{3}, c_{3}$, and $d_{3}$. As shown in Figure 6(b), $w_{32}$ and $w_{23}$ are the bottom lengths of $F C P_{3}$ and $F C P_{2}$, which are offset by $F C P_{2}$ and $F C P_{3}$, respectively. That is, $w_{32}$ and $w_{23}$ are $\overline{d_{3} P_{23}}$ and $\overline{P_{23} b_{2}}$, respectively. $w_{i j}$ can be estimated from the connection of two-sided FCPs, and $w_{i j}$ is the length of panel $i$ that is offset by panel $j$.

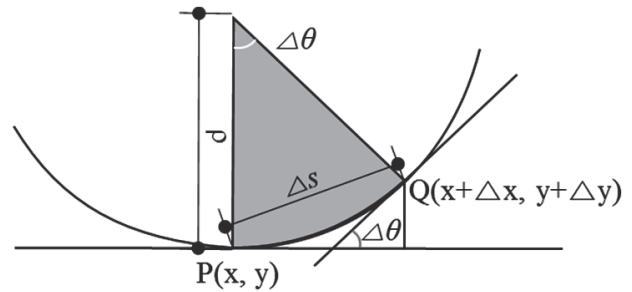

Figure 5. Curve adjustment

To estimate the offset volume, the angles of the FCPs that meet at different angles should be calculated. As indicated in Figure 3, $a_{1}$ means a corner edge at the lower part of FCP $P_{i}$ as shown in Figure 6(a). For example, $a_{1}, a_{2}$, and $a_{3}$ are defined as a corner edges at the lower part of $F C P_{1}, F C P_{2}$, and $F C P_{3}$, respectively. When connecting three FCPs, the parts between $a_{1}$ and $O$ ' for $F C P_{1}$, between $a_{2}$ and $O^{\prime}$ for $F C P_{2}$, and between $a_{3}$ and $O^{\prime}$ for $F C P_{3}$ should be offset with the same method applied for four FCPs, as shown in Figure 3. Thus, $a_{1}, a_{2}$, and $a_{3}$ should offset to connect each FCP corner to $O^{\prime}$.

$\theta_{1}$ is the angle consists of the bottom point of contact of $F C P_{1}$ and $F C P_{2}\left(P_{12}\right)$, the subordinate vertex $\left(O^{\prime}\right)$, and the bottom point of contact of $F C P_{3}$ and $F C P_{1}\left(P_{31}\right)$. That is, $\theta_{1}$ is $\angle P_{12} O^{\prime} P_{31}$. $\theta_{i}$ is defined as the angle of $F C P_{1}$ created when the corner edge of each offset FCP meets at $O$ '. $\theta_{12}$ is, in the initial-produced panel before $F C P_{1}$ is cut for installation, the angle created by the lower end point at the part meet with the $\mathrm{FCP}_{2}\left(b_{1}\right)$, a corner edge at the bottom part of $F C P_{1}\left(a_{1}\right)$, and the subordinate vertex $\left(O^{\prime}\right)$. That is, $\theta_{12}$ is $\angle b_{1} a_{1} O^{\prime}$. Additionally, $\theta_{13}$ is the angle created by the lower end point at the part meet with the $F C P_{3}\left(d_{1}\right)$, a corner edge at the bottom part of $F C P_{1}\left(a_{1}\right)$, and the subordinate vertex $\left(O^{\prime}\right)$. That is, $\theta_{13}$ is $\angle d_{1} a_{1} O^{\prime} . \theta_{i j}$ is defined as the angle of the corner edge for $F C P_{i}$ of the initial-produced panel before cutting.

Additionally, $\theta_{i j}$ is influenced by the connected FCP according to the FCP shape. In other words, $\theta_{i j}$ is the angle of panel $i$ impacted by panel $j$, which can be calculated using Eqn (4). $\theta_{13}$ can be calculated by dividing 'the value subtracted $\theta_{1}$ from $360^{\circ}$ ' by 'the value subtracted $\theta_{1}$ and $\theta_{3}$ from $360^{\circ}$, and multiplying $\theta_{1}$. Additionally, $\theta_{12}$ can be calculated by dividing 'the value subtracted $\theta_{1}$ from $360^{\circ}$, by 'the value subtracted $\theta_{1}$ and $\theta_{2}$ from $360^{\circ}$, and multi-

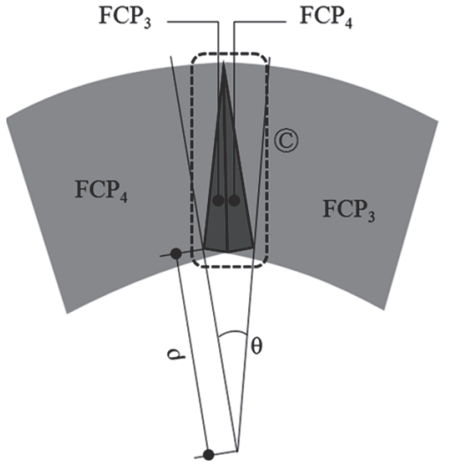

(a) Section (B) of offset edge

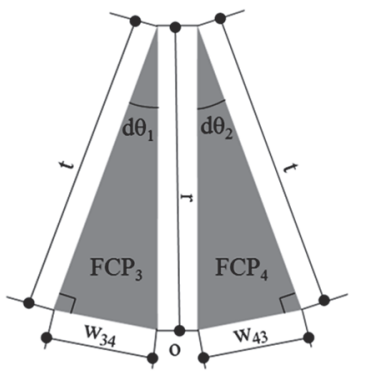

(b) Detail of (C)

Figure 4. Details of joints with FCPs 


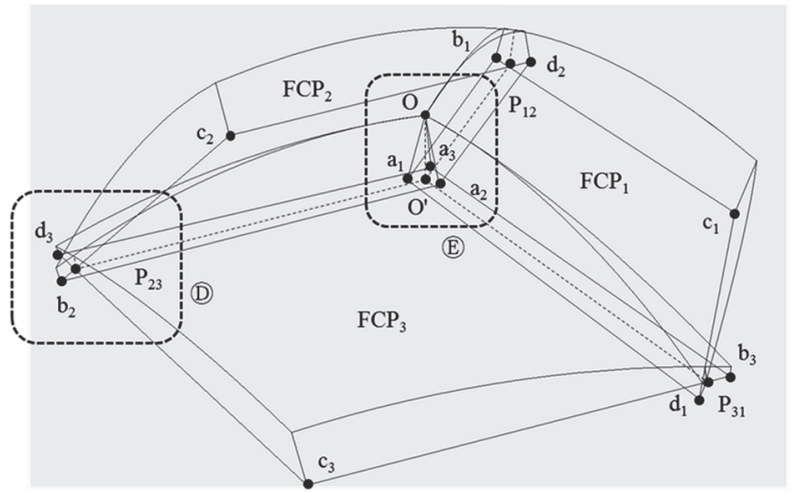

(a) Definition of point for three FCPs

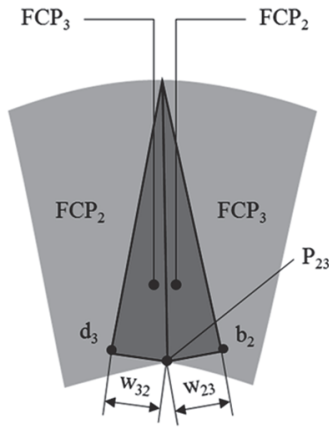

(b) Detail of cross section for (D)

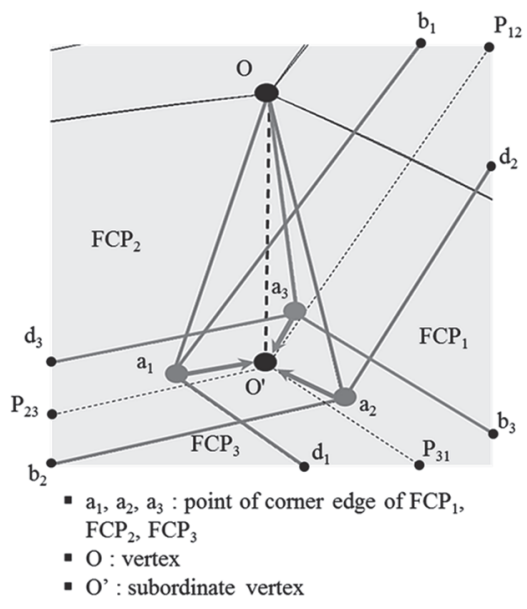

(c) Detail of (E)

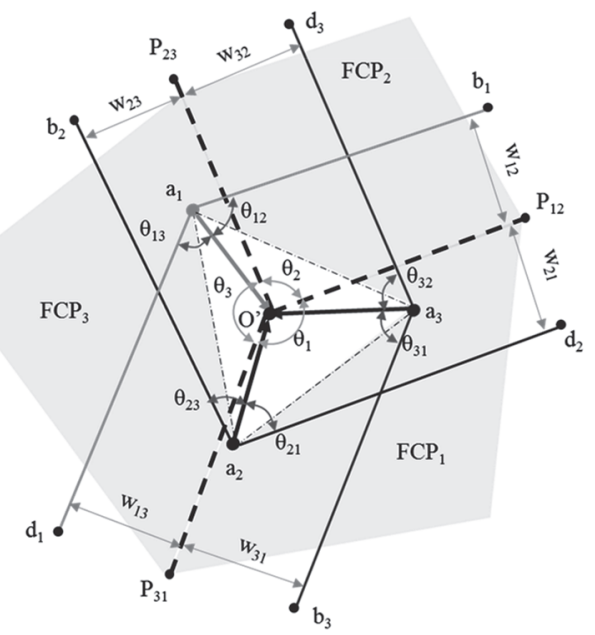

(d) Bottom plan view

Figure 6. Vertices with three FCPs

plying $\theta_{1}$. In the same manner, $\theta_{23}, \theta_{21}, \theta_{31}$, and $\theta_{32}$ can be calculated.

$$
\begin{aligned}
& \theta_{13}=\frac{360-\theta_{1}-\theta_{3}}{360-\theta_{1}} \cdot \theta_{1} ; \\
& \theta_{12}=\frac{360-\theta_{1}-\theta_{2}}{360-\theta_{1}} \cdot \theta_{1} ; \\
& \theta_{21}=\frac{360-\theta_{2}-\theta_{1}}{360-\theta_{2}} \cdot \theta_{2} ; \\
& \theta_{23}=\frac{360-\theta_{2}-\theta_{3}}{360-\theta_{2}} \cdot \theta_{2} ; \\
& \theta_{32}=\frac{360-\theta_{3}-\theta_{2}}{360-\theta_{3}} \cdot \theta_{3} ; \\
& \theta_{31}=\frac{360-\theta_{3}-\theta_{1}}{360-\theta_{3}} \cdot \theta_{3} .
\end{aligned}
$$

(2) Subordinate vertex with four-sided FCPs

The details associated with the subordinate vertex of a connection of four FCPs are shown in Figure 7. The lower section of an FCP with four connected sides is located as shown in Figure 7. Thus, $a_{1}, a_{2}, a_{3}$, and $a_{4}$ should offset to connect each corner of FCP to $O^{\prime}$. Accordingly, $w_{i j}$ can be calculated from the connection of two sides with the same method applied for the three FCPs. And $\theta_{i j}$ can be calculated using Eqn (5).

$\theta_{14}$ can be calculated by dividing the value subtracted $\theta_{1}$ from $360^{\circ}$ ' by 'the value subtracted $\theta_{1}$ and $\theta_{4}$ from $360^{\circ}$, and multiplying $\theta_{1}$. Additionally, $\theta_{12}$ can be calculated by dividing 'the value subtracted $\theta_{1}$ from $360^{\circ}$ ' by

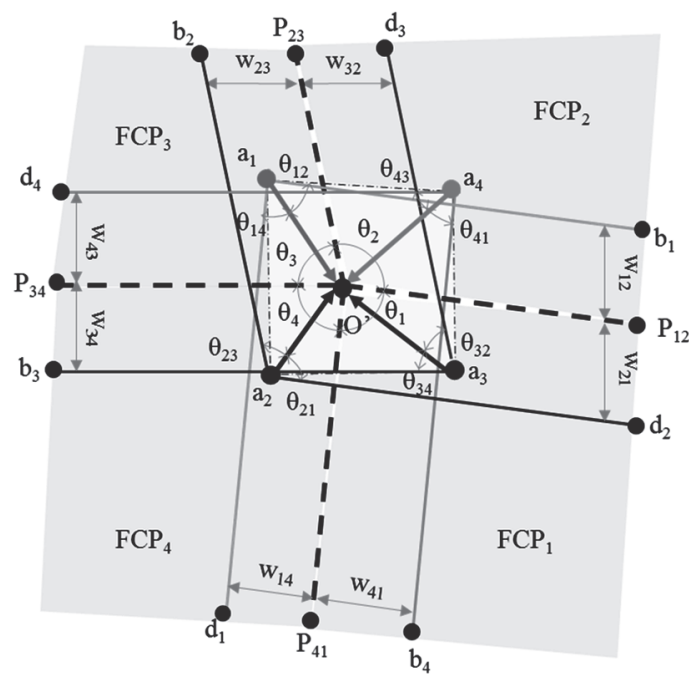

Figure 7. Bottom plan view of four FCPs 


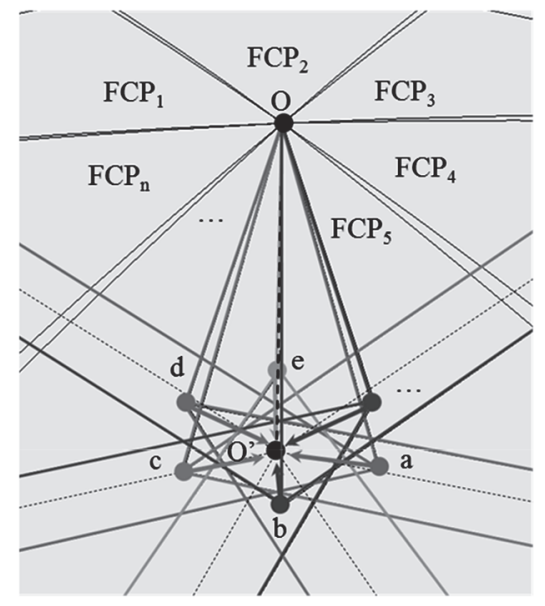

- $a_{1}, a_{2}, a_{3}, a_{4}, a_{5}, \ldots, a_{n}$ : point of corner edge of $\mathrm{FCP}_{1}, \mathrm{FCP}_{2}, \mathrm{FCP}_{3}, \mathrm{FCP}_{4}, \mathrm{FCP}_{5}$, $\ldots, \mathrm{FCP}_{\mathrm{n}}$

- $\mathrm{O}$ : vertex

- O' : subordinate vertex

(a) Detailed vertex with $\mathrm{n}$ FCPs

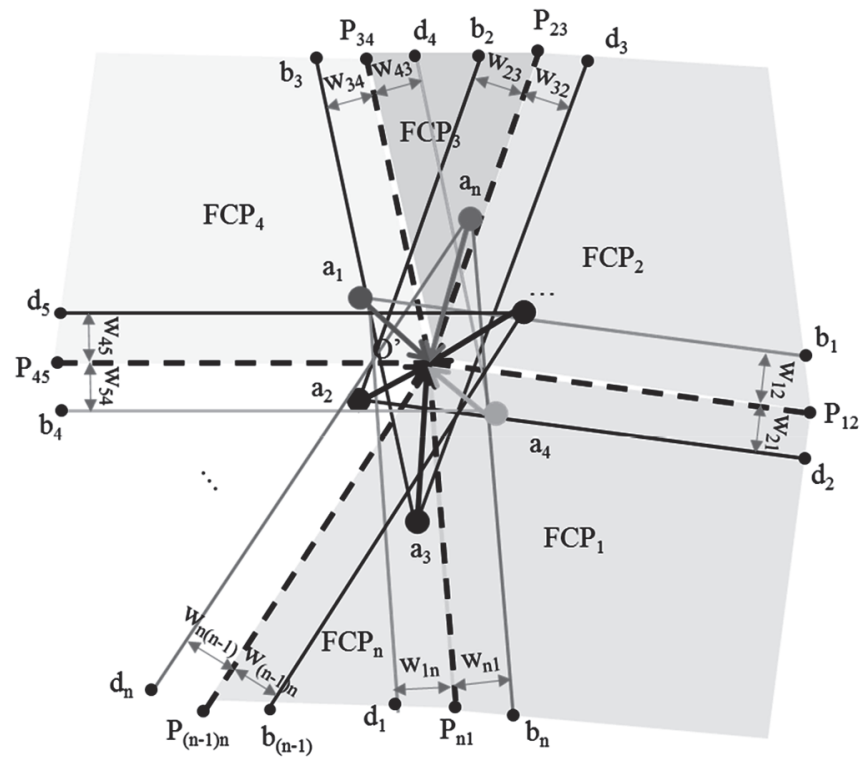

(b) Bottom plan view

Figure 8. Details of vertices with $n$-jointed FCPs

'the value subtracted $\theta_{1}$ and $\theta_{2}$ from $360^{\circ}$, and multiplying $\theta_{1}$. This is an equation of the same type as Eqn (4) applied in three FCPs. Additionally, $\theta_{21}, \theta_{23}, \theta_{32}, \theta_{34}, \theta_{43}$, and $\theta_{41}$ can be calculated in the same manner.

$$
\begin{aligned}
& \theta_{14}=\frac{360-\theta_{1}-\theta_{4}}{360-\theta_{1}} \cdot \theta_{1} ; \\
& \theta_{12}=\frac{360-\theta_{1}-\theta_{2}}{360-\theta_{1}} \cdot \theta_{1} ; \\
& \theta_{21}=\frac{360-\theta_{2}-\theta_{1}}{360-\theta_{2}} \cdot \theta_{2} ; \\
& \theta_{23}=\frac{360-\theta_{2}-\theta_{3}}{360-\theta_{2}} \cdot \theta_{2} ; \\
& \theta_{32}=\frac{360-\theta_{3}-\theta_{2}}{360-\theta_{3}} \cdot \theta_{3} ; \\
& \theta_{34}=\frac{360-\theta_{3}-\theta_{4}}{360-\theta_{3}} \cdot \theta_{3} ; \\
& \theta_{43}=\frac{360-\theta_{4}-\theta_{3}}{360-\theta_{4}} \cdot \theta_{4} ; \\
& \theta_{41}=\frac{360-\theta_{4}-\theta_{1}}{360-\theta_{4}} \cdot \theta_{4} .
\end{aligned}
$$

(3) Subordinate vertex with $n$-sided FCPs

When connecting $n$-sided FCPs, the lower section of the FCP is located as shown in Figure 8. Therefore, $a_{1}, a_{2}$, $a_{3}, a_{4}, a_{5}, \ldots, \mathrm{a}_{n}$ should offset to connect each corner of FCP to $O^{\prime}$. Accordingly, $w_{i j}$ can be calculated from the connection of two sides, and $\theta_{i j}$ can be estimated using Eqn (6).

$\theta_{1 n}$ can be calculated by dividing the value subtracted $\theta_{1}$ from $360^{\circ}$ ' by 'the value subtracted $\theta_{1}$ and $\theta_{n}$ from $360^{\circ}$, and multiplying $\theta_{1}$. Additionally, $\theta_{12}$ can be calculated by dividing 'the value subtracted $\theta_{1}$ from $360^{\circ}$ ' by 'the value subtracted $\theta_{1}$ and $\theta_{2}$ from $360^{\circ}$, and multiplying $\theta_{1}$. This is an equation of the same type as Eqn (4) and Eqn (5) applied in three FCPs and four FCPs. That is, as a result of deriving the angles equation for the point of contacts for $n$-FCPs, angle defined in the same manner has the same angle estimation equation regardless of the number of FCPs. Additionally, $\theta_{21}, \theta_{23}, \theta_{32}, \theta_{34}, \theta_{43}, \theta_{45}, \ldots, \theta_{(n)(n-1)}$, and $\theta_{n 1}$ can be calculated in the same manner.

$$
\begin{aligned}
& \theta_{1 n}=\frac{360-\theta_{1}-\theta_{n}}{360-\theta_{1}} \cdot \theta_{1} ; \\
& \theta_{12}=\frac{360-\theta_{1}-\theta_{2}}{360-\theta_{1}} \cdot \theta_{1} ; \\
& \theta_{21}=\frac{360-\theta_{2}-\theta_{1}}{360-\theta_{2}} \cdot \theta_{2} ; \\
& \theta_{23}=\frac{360-\theta_{2}-\theta_{3}}{360-\theta_{2}} \cdot \theta_{2} ; \\
& \theta_{32}=\frac{360-\theta_{3}-\theta_{2}}{360-\theta_{3}} \cdot \theta_{3} ; \\
& \theta_{34}=\frac{360-\theta_{3}-\theta_{4}}{360-\theta_{3}} \cdot \theta_{3} ; \\
& \theta_{43}=\frac{360-\theta_{4}-\theta_{3}}{360-\theta_{4}} \cdot \theta_{4} ; \\
& \theta_{45}=\frac{360-\theta_{4}-\theta_{5}}{360-\theta_{4}} \cdot \theta_{4} ; \\
& \theta_{(n)(n-1)}=\frac{360-\theta_{n}-\theta_{n-1}}{360-\theta_{n}} \cdot \theta_{n} ; \\
& \theta_{n 1}=\frac{360-\theta_{n}-\theta_{1}}{360-\theta_{n}} \cdot \theta_{n} .
\end{aligned}
$$




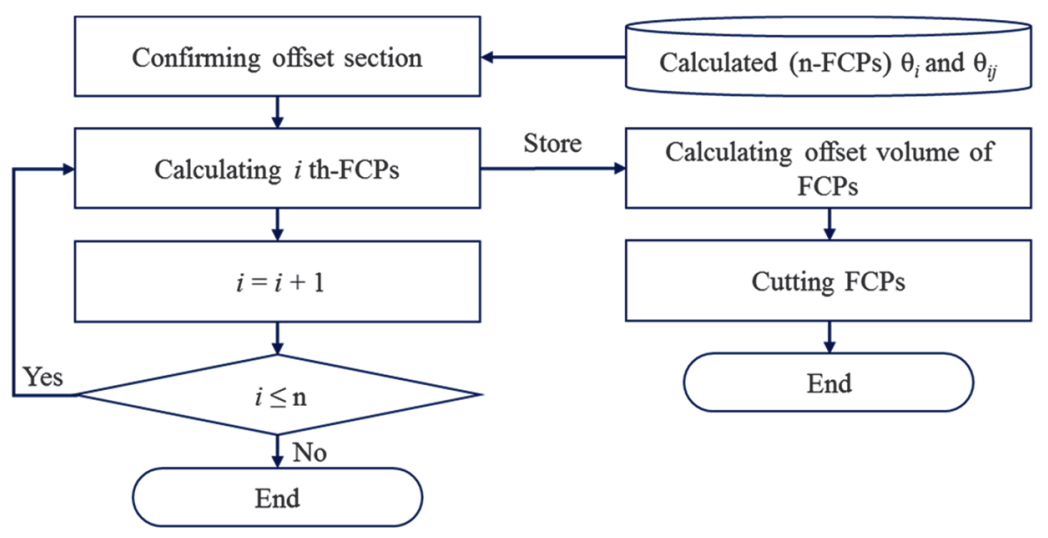

Figure 9. Offset volume estimation algorithm

The subordinate vertex can be controlled before installing external finish materials by calculating the joint length and angle when producing several FCPs. The equation proposed in this section to calculate the angle $\left(\theta_{i j}\right)$ and joint length $\left(w_{i j}\right)$ can be applied to control the subordinate vertex.

\subsection{Offset volume estimation algorithm}

If the angles of the $n$-FCPs for the subordinate vertex are estimated, calculating an offset volume for the angle is necessary. First of all, after confirming the offset section of FCPs, calculate the offset volume of $F C P_{1}$. Estimation of the offset volume is completed when the nth FCP is sequentially estimated. These values are stored and used for cutting after production of FCPs. The offset volume estimation algorithm is as shown in Figure 9.

The following assumption was made for accurate estimation of the offset volume needed to connect and precisely install two or more FCPs. The curvature of segments encountered at a vertex $(O)$ and subordinate vertex $\left(O^{\prime}\right)$ should be the same. Accordingly, FCPs should be produced considering the offset volume as shown in Figure 10.

The calculation of the offset volume based on the connection of three FCPs proposed in the study is as follows. The offset part of a side is shaped like a triangular prism, as shown in Figure 10(c), and the edge offset volume $\left(V_{A}\right)$ is calculated as shown in Eqn (7).

$$
V_{A}=\frac{1}{2} w_{A} \cdot t \cdot l_{A}
$$

where $w_{A}$ - offset width; $t$ - thickness, $l_{A}$ - edge length of an FCP.

The offset width $\left(w_{A}\right)$ is calculated with the same method applied for two FCPs in Figure 4. In other words, it is estimated with Eqn (8) using the offset angle $\left(\theta_{i j}\right)$ :

$$
w_{A}=t \cdot \tan \theta_{A} \text {. }
$$

$V B$ can be calculated using the same method. In addition, two offset sides are generated from a segment when three FCPs are connected, as shown in Figure 10(c). The corner edge with two offset parts overlaps. The offset volume of a corner edge can be accurately estimated when it is subtracted for calculation. As shown in Figure 10(c), the corner edge is shaped like a quadrangular pyramid, and it is calculated as shown in Eqn (9).

$$
V_{A} \cap V_{B}=\frac{1}{3} t \cdot w_{A} \cdot w_{B} \text {. }
$$

Thus, the total offset volume of each panel upon connection of three FCPs is as expressed in Eqn (10), and it can be accurately calculated. Additionally, the offset volume for the connection of $n$ number of FCPs can be estimated using the same mathematical algorithms:

$$
\begin{aligned}
& V=V_{A}+V_{B}-\left(V_{A} \cap V_{B}\right)= \\
& \frac{1}{2} w_{A} \cdot t \cdot l_{A}+\frac{1}{2} w_{B} \cdot t \cdot l_{B}-\frac{1}{3} \cdot t \cdot w_{A} \cdot w_{B}= \\
& \frac{1}{2} \tan \theta_{A} \cdot t \cdot l_{A}+\frac{1}{2} \tan \theta_{B} \cdot t \cdot l_{B}-\frac{1}{3} \cdot t \cdot \tan \theta_{A} \cdot \tan \theta_{B}= \\
& \frac{1}{2} \cdot t^{2}\left(\tan \theta_{A} \cdot l_{A}+\frac{1}{2} \tan \theta_{B} \cdot l_{B}\right)-\frac{1}{3} \cdot t \cdot \tan \theta_{A} \cdot \tan \theta_{B} .
\end{aligned}
$$

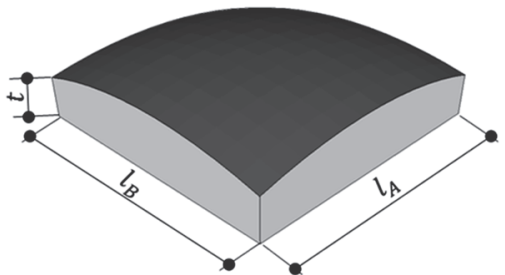

(a) Before offset

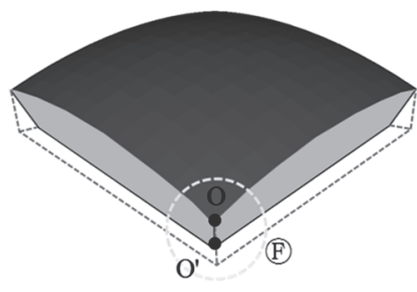

(b) After offset

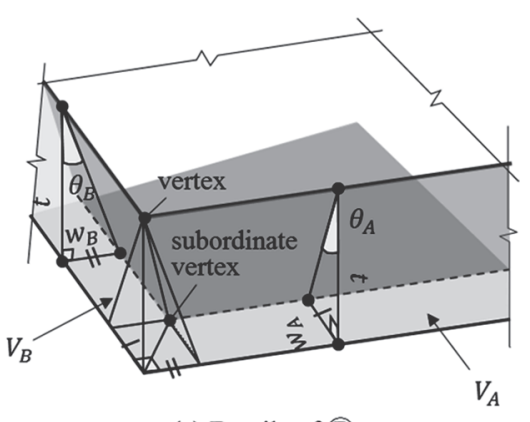

(c) Details of $\mathbb{P}$

Figure 10. Offset volume of FCPs 


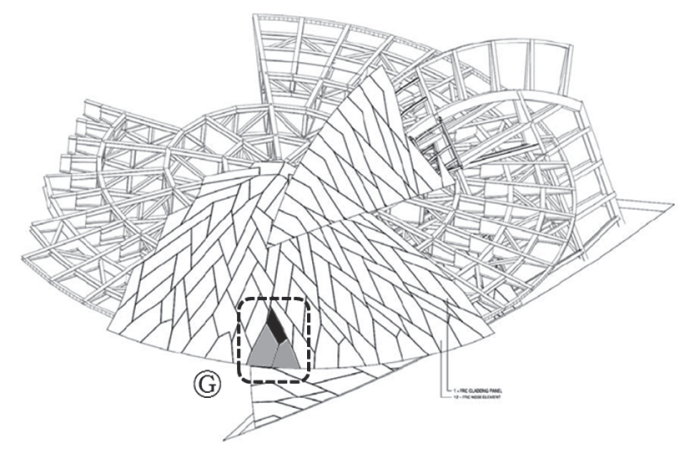

(a) Design sample in drawing

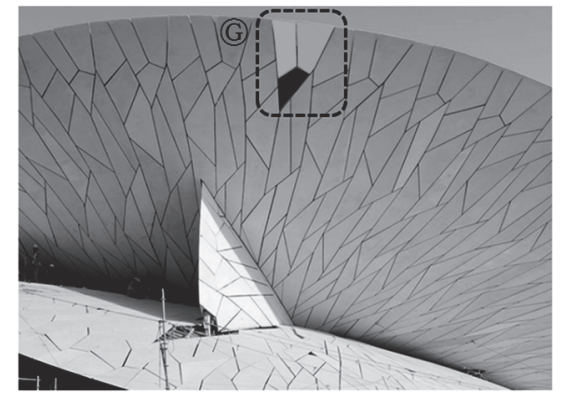

(b) Actual design on case project

Figure 11. Roof of Qatar national museum (Kim 2014)

When the section where three FCPs joint sides overlap is offset, the point of the corner edge shown in Figure 10(c) moves to the subordinate vertex, and the separation distance $(D)$ is calculated as shown in Eqn (11). This can be easily calculated using the offset width $\left(w_{i j}\right)$ calculated by Eqn (8).

$$
D_{n}=\sqrt{\left(w_{A}\right)^{2} \cdot\left(w_{B}\right)^{2}} .
$$

Thus, the mathematical algorithms derived in this study are applied to accurately calculate offset values (or geometry) of each panel at the junction (connection) point of three or more FCPs. Thus, it is possible to resolve inconsistencies in the panels' subordinate vertices upon FCP installation. That is, it is possible to produce FCPs for precise installation of free-form exterior panels.

\section{Case project application}

The equations for offset angle $\left(\theta_{i j}\right)$ and offset width $\left(w_{i j}\right)$ proposed in the study were applied to a case project. As shown in Figure 11, Qatar national museum was built using a combination of free-form concrete panels. However, it was constructed by considering the tolerance among panels because the issue of subordinate vertices was not resolved. In such a case, precise installation cannot be realized, and the aesthetic quality cannot be secured. Therefore, this case study is intended to estimate production information using a 3D modeling tool after calculating the offset values (or geometry) of each member from the FCPs' junction point using the proposed equations.

The formula for the subordinate vertex of this study is based on the following assumptions when panels are con- nected. First, the curvature of two segments is the same, and a point with the same curvature lies on a point of contact. Second, when the thickness and curvature of both panels are the same, $w_{1}=w_{2}$ (offset width) and $\theta_{1}=\theta_{2}$ (offset angle). Thus, in the case of (G) of Figure 11, the thickness of each panel is assumed to be $100 \mathrm{~mm}$, and the curvature of the two panels is the same. Figure 12 represents this case using a 3D modeling tool.

The formula for the subordinate vertex proposed in the study is used to calculate the offset values of each panel. The offset value of each panel is calculated as shown in Table 1 , and the offset part based on the estimated offset values is shown in Figure 12(b).

The process of estimating the offset values of $\mathrm{FCP}_{1^{-}}$ $\mathrm{FCP}_{2}$ is an example that provides a more specific explanation of the calculation process. It is assumed that the offset values $\left(\theta_{i j}\right)$ of $F C P_{1}-F C P_{2}, F C P_{2}-F C P_{3}$, and $F C P_{3}$ $F C P_{1}$ were $15^{\circ}, 12^{\circ}$, and $10^{\circ}$, respectively, and the corner lengths $\left(l_{i j}\right)$ were $1,500 \mathrm{~mm}, 1,000 \mathrm{~mm}$, and $1,000 \mathrm{~mm}$. The offset width $\left(w_{12}\right)$ and edge offset volume $\left(v_{12}\right)$ of $F C P_{1^{-}}$ $\mathrm{FCP}_{2}$ were calculated using Eqn (7) and Eqn (8), respectively, as shown below. The calculated values were rounded to the nearest tenth because the number that is displayed after the decimal point does not greatly impact the result. Additionally, the offset width $\left(w_{i j}\right)$ and edge offset volume $\left(v_{i j}\right)$ of $F C P_{2}-F C P_{3}$ and $F C P_{3}-F C P_{1}$ were calculated with the same method.

$$
\begin{aligned}
& w_{12}=t \cdot \tan \theta_{12}=100 \cdot \tan 15^{\circ}=27 \mathrm{~mm} ; \\
& V_{12}=\frac{1}{2} w_{12} \cdot t \cdot l_{12}=\frac{1}{2} \cdot 2.7 \cdot 10 \cdot 150=2,025 \mathrm{~cm}^{3} .
\end{aligned}
$$

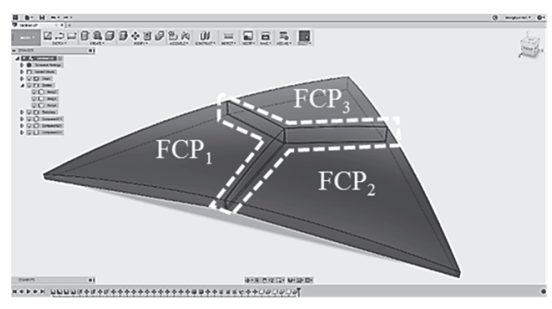

(a) 3D Modeling of (G)

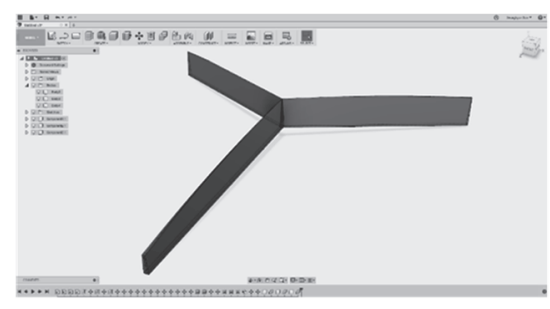

(b) Offset section of each FCP

Figure 12.3D modeling of roof panels (G) 
Table 1. Offset value of each panel

\begin{tabular}{|l|c|c|c|}
\hline \multicolumn{1}{|c|}{ Classification } & $F C P_{1}-F C P_{2}$ & $F C P_{2}-F C P_{3}$ & $F C P_{3}-F C P_{1}$ \\
\hline Offset angle $\left(\theta_{i j}\right)$ & $15^{\circ}$ & $12^{\circ}$ & $10^{\circ}$ \\
\hline Corner length $\left(l_{i j}\right)$ & $1,500 \mathrm{~mm}$ & $1,000 \mathrm{~mm}$ & $1,000 \mathrm{~mm}$ \\
\hline Offset width $\left(w_{i j}\right)$ & $27 \mathrm{~mm}$ each & $21 \mathrm{~mm} \mathrm{each}$ & $18 \mathrm{~mm}$ each \\
\hline Edge offset volume $\left(v_{i j}\right)$ & $2,025 \mathrm{~cm}^{3}$ & $1,050 \mathrm{~cm}^{3}$ & $900 \mathrm{~cm}^{3}$ \\
\hline
\end{tabular}

Table 2. Offset volume of each panel for production (unit: $\mathrm{cm}^{3}$ )

\begin{tabular}{|l|c|c|c|c|c|c|}
\hline \multicolumn{1}{|c|}{ Classification } & \multicolumn{2}{|c|}{$F C P_{1}$} & \multicolumn{2}{c|}{$F C P_{2}$} & \multicolumn{2}{c|}{$F C P_{3}$} \\
\hline \multirow{2}{*}{ Edge offset (a)= $V_{i j}$ ) } & $V_{12}$ & 2,025 & $V_{21}$ & 2,025 & $V_{31}$ & 900 \\
\cline { 2 - 6 } & $V_{13}$ & 900 & $V_{23}$ & 1,050 & $V_{32}$ & 1,050 \\
\hline Corner edge offset ((b) $\left.=V_{i j} \cap V_{j k}\right)$ & \multicolumn{2}{|c|}{16} & \multicolumn{2}{|c|}{19} & \multicolumn{2}{c|}{13} \\
\hline Total offset volume (a)-(b) & \multicolumn{2}{|c|}{2,909} & \multicolumn{2}{c|}{3,056} & \multicolumn{2}{c}{1,937} \\
\hline
\end{tabular}

Based on the estimated offset values, the corner edge and total offset volume for production of each panel were calculated using Eqn (9) and Eqn (10) as below. In addition, the offset volumes of $\mathrm{FCP}_{2}$ and $\mathrm{FCP}_{3}$ were calculated with the same method. The results of the calculations are shown in Table 2.

$V_{12} \cap V_{13}=\frac{1}{3} \cdot t \cdot w_{12} \cdot w_{13}=\frac{1}{3} \cdot 10 \cdot 2.7 \cdot 1.8=16 \mathrm{~cm}^{3} ;$

$V_{F C P_{1}}=V_{12}+V_{13}-\left(V_{12} \cap V_{13}\right)=2,025+900-16=2,909 \mathrm{~cm}^{3}$.

Thus, the offset volume of three-sided FCPs can be accurately calculated, and the separation distance $(d)$ of the point of corner edges that contact the subordinate vertex can be calculated using Eqn (10). Eqn (11) shows the process of estimating the point of corner edge for $F C P_{1}$, and the separation distance $(d)$ of the point of the corner edge for $F C P_{2}$ and $\mathrm{FCP}_{3}$ is calculated with the same method. The results of the calculations are shown in Table 3.

$$
d_{1}=\sqrt{\left(w_{12}\right)^{2}+\left(w_{13}\right)^{2}}=\sqrt{(27)^{2}+(18)^{2}}=32 \mathrm{~mm} . \text { (11) }
$$

According to the results shown in Table 3, the separation distances between the corner edge points for $F C P_{1}$, $\mathrm{FCP}_{2}$, and $\mathrm{FCP}_{3}$ and the subordinate vertex are $32 \mathrm{~mm}$, $34 \mathrm{~mm}$, and $28 \mathrm{~mm}$, respectively. Using these, the position of the subordinate can be expressed as accurate coordinates, and the offset volumes of $F C P_{1}, F C P_{2}$, and $F C P_{3}$ can be calculated. In other words, accurate dimensions and geometries that match the vertex at the upper FCP section and subordinate at the lower section are obtained.

Thus, the formula for subordinate vertices can be used to easily and quickly calculate the offset values or geometry prior to FCP design. The formula can accurately deduct the position of the subordinate vertex of each panel and the offset volume and address overlap upon erection and installation of FCPs. That is, finish pattern between panels can be accurately expressed, securing the aesthetic quality of free-form buildings. Additionally, time and manpower required for construction of free-form buildings can be re-
Table 3. Distance between corner edge and subordinate vertex

\begin{tabular}{|c|c|c|c|}
\hline Classification & $F C P_{1}$ & $F P_{2}$ & $F C P_{3}$ \\
\hline $\begin{array}{l}\text { Separation distance of the } \\
\text { point of corner edge }\left(\mathrm{D}_{n}\right)\end{array}$ & $32 \mathrm{~mm}$ & $34 \mathrm{~mm}$ & $28 \mathrm{~mm}$ \\
\hline
\end{tabular}

duced as the problems related to subordinate vertices upon FCP production can be controlled in advance.

Construction accuracy upon FCP installation was secured using the equations derived for estimating angles of vertices and offset volume. When this method is used for panel production, it is expected that the erection and installation of FCPs will be easier, resulting in improved productivity, reduced time, and reduced cost.

\section{Conclusions}

Various types of free-form buildings are being constructed due to the development of computer science, materials, and equipment as design and construction technologies are enhanced. Free-form building design and construction involve problems such as decreased productivity and increased cost and construction time. When concrete panels are chosen for FCP production, the largest problem is inconsistencies in the subordinate vertices upon FCP installation. To solve this problem, the formula for subordinate vertices and equations for calculating offset volume and separation distance of corner edges based on the number of junction points were deduced. The equations were applied to FCPs produced for actual construction at Qatar national museum to verify their usability. The conclusions drawn from the study are as follows.

First, it is derived equations for calculating the angles of the subordinate vertex to solve the problem of offsets between FCPs. Using these, it was confirmed that the angles defined at the point of contacts for n-FCPs have angle equations of same type irrespective of the number of FCPs. Additionally, the equation was applied for a case where three FCPs are in contact, and the offset angles of 
$F C P_{1}, F C P_{2}$, and $F C P_{3}$ were calculated to be $15^{\circ}, 12^{\circ}$, and $10^{\circ}$, respectively. Through this application, the proposed equations are generalizable and applicable to all FCPs with multiple sides in connection (junction). This is possible because the developed equation considers the fact that subordinate vertices increased and consist of a more complicated form when three or more free-form panels meet. It can easily and quickly calculate the angle using the exact equations derived from this study, and this values can be used to calculate the offset volume to reduce the FCPs production time.

Second, when three FCPs are in contact, equations for calculating offset volume and separation distance of a corner edge were applied. As a result, the total offset volumes of $F C P_{1}, F C P_{2}$, and $F C P_{3}$ were $2,909 \mathrm{~cm}^{3}, 3,056 \mathrm{~cm}^{3}$, and $1,937 \mathrm{~cm}^{3}$, respectively. In addition, the separation distances of the corner edge were calculated to be $32 \mathrm{~mm}$, $34 \mathrm{~mm}$, and $28 \mathrm{~mm}$, respectively. The equations verified that it is possible to accurately calculate the separation distance of corner edges and the volume of offset joints, even when FCPs are encountered at different angles. In other words, precise installation of free-form panels is possible by accurate cutting the edge surface of the FCPs using the offset volume formula.

Third, the sides of FCPs that need to be offset for accurate connections can be calculated using the equations proposed in the study. Thus, FCPs can be produced for easier erection and installation on-site. Additionally, an aesthetic finish pattern can be realized since the quality of free-form buildings can be secured as the subordinate vertex problem is resolved using BIM.

In this study, it is confirmed that the problem of the subordinate vertices of FCPs can be solved using angle calculation formulas and the offset volume formula of the vertex developed in the FCPs manufacturing phase. It is expected that time and manpower will be reduced when constructing free-form buildings. The formulas developed through this study will support the production of FCPs, resulting in high productivity and precision in practice, and the required time will also be shortened. This paper academically contributes to the automatic creation of joint details of FCPs implemented by BIM and it will be used for the study solving the subordinate vertices problem by mathematical algorithm.

In general, free-form means a form that is not defined by mathematical functions. However, in the case of a building made up of 3-dimensional curved panels of even it that can be defined by mathematical expressions, the process of manufacturing and installing of each panel is difficult because it is similar to the level of free-form building. The Qatar national museum, selected as a case project in this study, also corresponds to this case. This is a study to solve the problem that can be defined by mathematical equations although it is difficult to manufacture and install to similar to the level of free-form panels. Therefore, additional research is necessary for free-form building, which is difficult to define by mathematical formulas.

\section{Acknowledgements}

This work was supported by the National Research Foundation of Korea (NRF) grant funded by the Korea government (MOE) (No. 2017R1D1A1B04033761).

\section{References}

Bae, K. J.; Lee, S. H.; Jun, H. J. 2009. A study on digital design process of the materialization of free form design architecture, in 2009 Autumn Annual Conference of the Architectural Institute of Korea 29(1): 221-224.

Daniel, J. I.; Shah, S. P. (Eds.). 1990. Thin-section fiber reinforced concrete and ferrocement. ACI Special Publication SP-124.

Jovanovic, M.; Rakovi, M.; Tepavcevic, B.; Borovac, B.; Nicolic, M. 2017. Robotic fabrication of freeform foam structures with quadrilateral and puzzle shaped panels, Automation in Construction 74: 28-38. https://doi.org/10.1016/j.autcon.2016.11.003

Keneti, A. R.; Jafari, A.; Wu, J. H. 2008. A new algorithm to identify contact patterns between convex blocks for threedimensional discontinuous deformation analysis, Computers and Geotechnics 35(5): 746-759.

https://doi.org/10.1016/j.compgeo.2007.12.002

Kim, G. B.; Pilakoutas, K.; Waldron, P. 2008. Development of thin FRP reinforced GFRC permanent formwork systems, Construction and Building Materials 22: 2250-2259. https://doi.org/10.1016/j.conbuildmat.2007.07.029

Kim, H. 2010. A study on change of form design in modern high rise building architecture: Master's thesis. Incheon National University, Republic of Korea.

Kim, K. 2014. Development of production and management technology of free-form concrete segment: Master's thesis. Kyung Hee University of Architectural Engineering.

Kim, K.; Son, K.; Kim, E. D.; Kim, S. 2015. Current trends and future directions of free-form building technology, Architectural Science Review 58(3): 230-243. https://doi.org/10.1080/00038628.2014.927751

Kulovec, S.; Kos, L.; Duhovnik, J. 2012. Global minimization of vertex height differences for freeform architectural design, Journal of Software Engineering and Applications 5(9): 659-663. https://doi.org/10.4236/jsea.2012.59077

Lee, D.; Lim; J.; Habimana, G.; Lee, T. O.; Kim, S. 2015. Automatic panelizing algorithms of free-form buildings, in The $6^{\text {th }}$ International Conference on Construction Engineering and Project Management (ICCEPM 2015), Korea Institute of Construction Engineering and Management, 425-428.

Li, Y.; Zhang, E.; Kobayashi, Y.; Wonka, P. 2010. Editing operations for irregular vertices in triangle meshes, ACM Transactions on Graphics (TOG) 29(6): 153. https://doi.org/10.1145/1866158.1866179

Lim, J.; Kim, D. Y.; Kim, S. 2018. An experimental study for quality assurance of free-form concrete panels produced by CNC machine, Journal of Civil Engineering and Management 24(2): 150-159. https://doi.org/10.3846/jcem.2018.461

Lyu, H. C. 2009. Characteristics of digital tectonics of free-form structures designed through digital process, Journal of Korea Society of Design Forum, 225-236. https://doi.org/10.21326/ksdt.2009.25.021

Peled, A.; Shah, S. P.; Banthia, N. (Eds.). 2000. High-performance fiber-reinforced concrete thin sheet products. American Concrete Institute.

Precast/Pre-stressed Concrete Institute. 2001. Concrete Institute, Recommended practice glass fiber reinforced concrete 
panels. PCI Committee on Glass Fiber Reinforced Concrete Panels.

Reda, M. M.; Shrive, N. G.; Gillott, J. E. 1999. Microstructural investigation of innovative UHPC, Cement and Concrete Research 29(3): 323-329. https://doi.org/10.1016/S0008-8846(98)00225-7

Ryu, H. G.; Kim, S. J. 2012. Case study of concrete surface design and construction method for freeform building based on BIM: focused on Tri-Bowl, Korea, Journal of the Korea Institute of Building Construction 12(3): 347-357. https://doi.org/10.5345/JKIBC.2012.12.3.347

Ryu, H. K.; Kim, S. J. 2013. CNC twisted tube method for 3D coordinate control technology for freeform structure - focused on the ARC in DaeGu, Journal of the Korea Institute of Building Construction 13(5): 434-440. https://doi.org/10.5345/JKIBC.2013.13.5.434

Ryu, J. W. 2012. BIM-based panelizing optimization for architectural freeform surfaces: Doctor's thesis. Seoul National University of Architectural Engineering, Republic of Korea.

Sacks, R.; Eastman, C. M.; Lee, G. 2004. Parametric 3D modeling in building construction with examples from precast concrete, Automation in Construction 13(3): 291-312. https://doi.org/10.1016/S0926-5805(03)00043-8

Son, S.; Lim, J.; Kim, S. 2018. Planning algorithms for in-situ production of free-form concrete panels, Automation in Construction 91: 83-91.

https://doi.org/10.1016/j.autcon.2018.03.006 